\title{
Deux disques de musique du Mozambique
}

\section{Moira Laffranchini}

\section{OpenEdition}

\section{Journals}

Édition électronique

URL : http://journals.openedition.org/ethnomusicologie/942

ISSN : 2235-7688

Éditeur

ADEM - Ateliers d'ethnomusicologie

Édition imprimée

Date de publication : 1 décembre 1997

Pagination : 355-357

ISBN : 2-8257-0579-9

ISSN : 1662-372X

Référence électronique

Moira Laffranchini, «Deux disques de musique du Mozambique », Cahiers d'ethnomusicologie [En ligne], 10 | 1997, mis en ligne le 06 janvier 2012, consulté le 22 avril 2019. URL : http:// journals.openedition.org/ethnomusicologie/942

Ce document a été généré automatiquement le 22 avril 2019.

Tous droits réservés 


\title{
Deux disques de musique du Mozambique
}

\author{
Moira Laffranchini
}

\section{RÉFÉRENCE}

Ilha de Moçambique. Danze e canti della costa settentrionale. Danças e cantos da costa setentrional. Enregistrements (1995) : Alessandro Rossini, Erasmo Treglia ; texte et photographies :

Erasmo Treglia. Livret de 16 pages en italien et portugais, resumé en anglais et français, 3 photographies, 14 plages. 1 CD Crocevia/SudNord Records SN0040, 1995. Durée totale : 57' 75" (coll. « FOLKlore 7, Musiche dal nuovo mondo, Mozambico »).

Arcos, cordas, flautas. Strumenti musicali del Centro e del Sud. Instrumentos musicais do Centro e do Sul. Enregistrements (1993) : Lucas J. Mucavele, João A. Vilanculo; texte : Erasmo Treglia, João A. Vilanculo. Livret de 16 pages en italien et portugais, resumé en anglais et français, illustration des instruments, 20 plages. 1 CD Crocevia/SudNord Records SN0041, 1995. Durée totale : 62' 13” (coll. « FOLKlore 8, Musiche dal nuovo mondo, Mozambico »).

1 Ancienne capitale du royaume du Mozambique, l'Ile de Mozambique est un lieu historique de métissage culturel de grand intérêt. Se trouvant sur la route commerciale de l'or, de l'ivoire, des épices et des esclaves de la côte orientale de l'Afrique, les populations locales d'origine bantoue ont, au fil des siècles, subi les influences d'autres cultures, en particulier celle des Arabes, mais aussi celles de l'Inde et de l'Occident. Elles se manifestent dans la langue, dans les pratiques sociales, dans les cérémonies et les fêtes et, bien sûr, dans la vie quotidienne des habitants de l'île. Ce processus de synthèse concerne naturellement la musique et la danse, déterminant le développement de styles et de répertoires qui, même si on les retrouve dans la région côtière septentrionale du Mozambique, revêtent sur l'Ile de Mozambique un important degré d'originalité et d'intérêt. La première publication se veut une anthologie des différents répertoires musicaux présents sur l'île. 
2 Le tufo (plages 1, 5, 11)est un genre musical et une danse; il est le plus important et le plus connu de l'île, il peut aussi être admiré sur la côte des provinces de Nampula et de Cabo Delgado. Le répertoire du tufo est exécuté par deux chanteuses solistes et un chœur féminin accompagnés par une section rythmique d'instruments à percussion joués exclusivement par les hommes. La danse "sur place» est exécutée par le chœur, qui maintient le rythme en frappant des pieds tout en effectuant des mouvements synchronisés de flexion ou d'élévation du corps, de la tête, des bras et du tronc dirigés par le groupe des percussions. Généralement, on utilise quatre tambours sur cadre de différentes dimensions, à une membrane en peau d'antilope. Ces instruments peuvent être ronds, carrés, hexagonaux ou octogonaux. La présence d'éléments culturels arabes se remarque aisément, tant sur le plan mélodique que sur celui des instruments et surtout de la langue, qui peut être le naharra (dialecte makua de l'île), le swahili ou l'arabe.

D’autres ensembles de l'île sont spécialisés dans le répertoire du nsope (plages 4, 7, 13), genre musical très proche du tufo, chanté aussi par deux solistes et un chœur féminin accompagnés d'une section d'instruments à percussion. Nsope est littéralement la « danse de la corde » car une ou plusieurs danseuses sautent en alternance par dessus la corde qui se déroule devant le chœur et les musiciens. Cette danse peut également être réalisée avec une chorégraphie circulaire réunissant un nombre plus élevé de danseuses.

$4 \quad$ Le tufo et le nsope sont exclusivement des musiques de danse. Les tambours sont joués seulement par les hommes, contrairement à d'autres percussions, comme des barres de métal ou les hochets, qui peuvent être joués aussi par les femmes. L'esprit compétitif des différents groupes suscite une continuelle recherche de nouveauté, mais les nouveaux textes, liés aux thématiques de la vie quotidienne ou de la vie politique, sont composés en respectant le style musical.

5 Maulide (plages 3 et 10) est le nom d'une danse de type fakirique présente au nord du Mozambique ainsi que dans d'autres régions d'Afrique orientale. Il s'agit d'une danse rituelle islamique marquant la célébration de la naissance du Prophète et pratiquée par des hommes appartenant à une confrérie Le chant et la danse y sont accompagnés du seul rythme des tambours.

6 Les chants des pêcheurs (plages $6,8,12$ ) sont des chants de travail accompagnant les différentes étapes de la pêche. Deux voix solistes chantent une mélodie principale à laquelle répond le groupe de pêcheurs. Une autre partie du répertoire est en revanche formée de chants plus libres du point de vue rythmique et mélodique. Ces derniers accompagnent des activités de préparation à la pêche, comme la réparation des filets, ou simplement des moments de convivialité et de repos.

7 L'école coranique Madrasah Al Quran (Pemba) (plages 2, 9, 14) enseigne des chants qui font partie intégrante de la formation religieuse et culturelle des élèves.ll s'agit de chants pour des cérémonies religieuses et d'autres à caractère plus profane exécutés lors de manifestations publiques.

8 Le second $\mathrm{CD}$ est dédié à certains instruments traditionnels joués dans le centre et le sud du Mozambique comme le xitende (plages 2, 7, 10, 14, 17, 20), un arc musical d'environ deux mètres de long, tendu d'une corde métallique frappée avec une baguette en bois et pourvu d'une calebasse fixée au centre de l'arc. C'est un instrument joué en solo, mais il peut aussi accompagner le chant, qui est normalement de type récitatif ; il est souvent joué par un homme qui a l'intention de se marier et fait ainsi savoir aux parents des filles en âge de mariage qu'il est disponible. Le xitende est également l'instrument typique des 
musiciens ambulants qui parcourent les villes en chantant les principales étapes de l'histoire du Mozambique.

9 Le xizambi (plages $1,4,8,13$ ) est un arc musical en bois tendu d'une large corde en feuille de palmier. Sa particularité réside dans le fait que le son est obtenu en frottant avec une baguette non pas la corde, mais des incisions pratiqués sur l'arc en utilisant la bouche du musicien comme résonateur. Le xizambi est aussi connu sous les noms de xizambiza, chivelane, chinyacazembe et nyakazeze. Cet instrument est généralement lié au monde pastoral et les chants qu'il accompagne parlent du travail quotidien, mais aussi de morale ou d'arguments plus satiriques.

Dans le chivonkovoko (plages 3 et 11), l'arc passe à travers la caisse de résonance faite d'une noix de coco coupée sur un côté et recouverte d'une membrane de peau animale ; la corde est une racine enduite de résine végétale. Il peut être joué en solo ou en orchestre avec d'autres instruments.

11 Le nyanga (plages 9 et 15) est une flûte de Pan composée d'une rangée de tuyaux de bambou de différentes longueurs, fermés à leur extrémité inférieure. Le nombre des tuyaux varie entre deux et sept, et trois registres sont prévus. Le nyanga est joué en orchestre et, en même temps, les musiciens chantent et dansent en cercle. Les textes sont liés à l'occasion pour laquelle on danse : funérailles, célébration ou fête.

Le xingoviya (plages $5,12,18$ ) est une flûte globulaire fabriquée à partir d'un fruit séché ou façonnée en terre cuite. Le musicien souffle dans le trou central, tandis que deux trous latéraux servent à moduler le son. L'instrument est généralement joué en duo par les bergers, mais les filles l'utilisent volontiers pour accompagner leurs chants. Il est aussi connu sous le nom de xiphuwewe, goringo ou guerure.

13 Le nsansy (plages 6 et 16) est un lamellophone plutôt connu à l'étranger sous le nom de mbira. Les exemple de cette anthologie proviennent de la province de Tete. Enfin, la plage 19 est enfin dédiée à un morceau de chant et guitare.

14 Cette anthologie constitue un travail intéressant surtout grâce à la qualité de l'exécution et au niveau artistique des différents musiciens, tous d'âge assez avancé et de grande expérience. La publication de musiques traditionnelles mozambicaines étant encore limitée, ces enregistrements deviennent précieux dans une optique de sauvegarde du patrimoine culturel. Cependant, on peut regretter que, en voulant réaliser une anthologie, ce qui est peut-être nécessaire dans le paysage ethnomusicologique mozambicain, les auteurs aient privilégié une approche quantitative au détriment d'une présentation systématique des différents instruments, musiques et danses du Mozambique. Signalons encore la publication d'une brochure de Vasco Jacinto Nhussi concernant les deux CD sous le titre «A música tradicional de Moçambique: antologias das canções ») édition de l'ARPAC, collection Embondeiro n 9, Maputo, Juillet 1996). 